\title{
Localization of Elko spinor fields in tachyonic de Sitter braneworld models
}

\author{
Masoumeh Moazzen Sorkhi ${ }^{\mathrm{a}}$, Zahra Ghalenovi \\ Department of Physics, Faculty of Basic Sciences, Kosar University of Bojnord, Bojnord, Iran
}

Received: 30 November 2019 / Accepted: 12 March 2020 / Published online: 8 April 2020

(C) The Author(s) 2020

\begin{abstract}
In this paper, we investigate the localization of a five-dimensional $(5 D)$ Elko spinor field in de Sitter tachyonic braneworld models. These branes are generated by gravity coupled to a tachyonic bulk scalar field and include a de Sitter cosmological background induced on the $3 D$-brane. Using Yukawa-type coupling mechanism we show that a free massless Elko spinor field cannot be localized on tachyonic de Sitter branes while massive Elko fields are localized if their bulk mass obeys an upper bound. Also by introducing a tachyonic function $\mathrm{F}(\mathrm{T})$ as Yukawa interaction term between the Elko spinor and the background tachyonic scalar field we find that localized massless zero modes are given in terms of general Heun functions. Furthermore, it is shown that employing a new derivative coupling term in the action of Elko spinor fields leads to the Elko field localization on tachyonic de Sitter branes.
\end{abstract}

\section{Introduction}

It is well known that Extra dimensions and braneworld models can provide new insights to solve some problems in new physics, such as cosmological constant problems [1], Casimir force [2] and the gauge hierarchy problem [3]. In the Randall and Sundrum (R-S) model [4,5], the extra dimension does not need to be compactified and the brane is assumed very thin. This thin brane does not provide a smallest scale in a fundamental theory hence realistic branes are needed to have thickness and inner structure. In order to avoid the use of singular R-S brane, thick braneworld models have been proposed as a smooth generalization of the $\mathrm{R}-\mathrm{S}$ one $[6,7]$. In this scenario, the brane is usually generated by a bulk scalar field with kink configuration by a specific choice of the scalar potential. Various kinds of bulk scalar fields lead to different sorts of braneworld models can be found in Refs. [8-12]. In this framework, tachyonic braneworld models generated

a e-mail: m.m.sorkhi@kub.ac.ir (corresponding author) by a tachyon scalar field were introduced with applications to cosmology and supergravity $[13,14]$. In Ref. [15] it was shown that these tachyonic branes have several remarkable properties such as a regular and stable solution that contains a relevant $3 D$-brane with de Sitter $(\mathrm{dS})$ induced metric in a higher dimensional manifold that are interesting from the point of view of cosmology. Furthermore, the authors of Ref. [16] indicated that this tachyonic braneworld allows for a nontrivial solution with a vanishing $5 \mathrm{D}$ cosmological constant.

In the context of the braneworld physics, gravity and various bulk matter fields must be confined on a brane by a natural mechanism in order to recover our $4 D$ Universe and Standard Model of particle physics. In more studies [5, 17$21]$, it was found that graviton and massless scalar fields can be localized on branes of different types. In order to trap vector and tensor gauge fields in the R-S brane or its generalization thick braneworld models some new mechanisms are needed [12,22-25]. Fermions can be also localized on branes in five dimensions by introducing a Yukawa coupling term between fermions and background scalar field which is an odd function of extra dimension [26,27]. In Ref. [28], the authors have investigated a new fermion localization mechanism which employed a derivative fermion-scalar coupling term for which the background scalar field can be an odd or even function of the extra dimension. There are a lot of works that study the matter fields localization in braneworld models. For some comprehensive reviews, see Refs. [29-34].

Recently, a new spin-1/2 quantum field beyond the Standard Model proposed by the authors of Ref. [35] that well known as the Elko spinor field. An Elko field which is the eigenspinor of the charge conjugation operator with one mass dimension can be considered as a candidate of dark matter $[36,37]$. Also, localization of five-dimensional Elko spinor fields can be achieved in Minkowski thick branes by Yukawatype coupling mechanism [38-40] and non-minimal coupling mechanism [41]. Furthermore, it was found in Refs. [42-44] 
that the zero mode of Elko spinor field can be localized on de Sitter (dS) and Anti-de Sitter (AdS) branes with both mentioned mechanisms.

However, localization of Standard Model matter fields and gravity except Elko field in de Sitter tachyonic braneworld models have been considered in Refs. [15,31,45,46]. In this paper, we are interested to analyze several aspects of localization of the Elko spinor field on these branes. By using the Yukawa coupling term between the background scalar field and Elko spinor, we will show that the zero mode of Elko fields can be localized on de Sitter tachyonic branes. Also, by introducing a new derivative coupling term in $5 \mathrm{D}$ Elko field action we will investigate the localization of Elko spinor field.

The plan of this paper is as follows. In Sect. 2, we briefly summarize the de Sitter tachyonic braneworld models. In Sect. 3, we study the localization of the Elko spinor fields on this brane by the Yukawa coupling mechanism and investigate the localization of massless and massive Elko spinor fields. In Sect. 4, we investigate the Elko spinor field localization mechanism by employing a derivative coupling term. Finally, a summary and conclusion are given in Sect. 5 .

\section{The de Sitter tachyonic brane}

In this section, we review a de Sitter tachyonic thick brane setup constructed by gravity and a bulk tachyonic scalar field in the presence of a $5 \mathrm{D}$ cosmological constant. The metric is given by

$d s^{2}=e^{2 A(w)}\left[-d t^{2}+a^{2}(t)\left(d x^{2}+d y^{2}+d z^{2}\right)+d w^{2}\right]$,

where $e^{2 A(\omega)}$ and $a(t)$ are the warp factor and the scale factor of the brane, respectively. Also, $w$ shows the extra dimensional coordinate. In order to construct the de Sitter tachyonic brane solution, we start with the $5 D$ following action for a bulk tachyonic scalar field $T$ which coupled to gravity as [15],

$$
\begin{aligned}
S= & \int d^{5} x \sqrt{-g}\left[\frac{1}{2 \kappa_{5}^{2}} R-\Lambda_{5}\right. \\
& \left.-V(T) \sqrt{1+g^{M N} \partial_{M} T \partial^{N} T}\right],
\end{aligned}
$$

where $R$ is the five-dimensional scalar curvature and $\Lambda_{5}$ is the bulk cosmological constant and $M, N=0,1,2,3,5$. The $\kappa_{5}^{2}=4 \pi G_{5}$ where the $G_{5}$ is the five-dimensional Newton constant. We also notice that such real tachyonic scalar field $T$ depends only on the extra dimension $w$ and $V(T)$ is its self-interaction potential. The matter field equation of motion resulting from the action (2) is

$\partial_{M}\left[\frac{\sqrt{-g} V(T) \partial^{M} T}{\sqrt{1+(\nabla T)^{2}}}\right]-\sqrt{-g} \sqrt{1+(\nabla T)^{2}} \frac{\partial V(T)}{\partial T}=0$.

Using the metric ansatz (1) one can obtain following complete solutions [15]

$$
\begin{aligned}
& a(t)=e^{H t} \quad A(w)=\frac{1}{2} \ln [\operatorname{ssech}(H(2 w+c))], \\
& T(w)= \pm \sqrt{\frac{-3}{2 \kappa_{5}^{2} \Lambda_{5}}} \arctan \left(\frac{\sinh \left[\frac{(H(2 w+c))}{2}\right]}{\sqrt{\cosh [(H(2 w+c))]}}\right) \\
& = \pm b \operatorname{arcsinh}(\tanh (H w)) \text {, } \\
& V(T)=-\Lambda_{5} \sqrt{(1+\operatorname{sech}[H(2 w+c)])\left(1+\frac{3}{2} \operatorname{sech}[H(2 w+c)]\right)} \text {, }
\end{aligned}
$$

where $\mathrm{H}, \mathrm{c}$ and $\mathbf{s}$ are arbitrary positive constants while the $5 \mathrm{D}$ cosmological constant $\Lambda_{5}$ is negative. Also, the constants $\mathbf{S}$ and $b$ are defined by

$b=\sqrt{\frac{-3}{2 \kappa_{5}^{2} \Lambda_{5}}} \mathbf{s}=-\frac{6 H^{2}}{\kappa_{5}^{2} \Lambda_{5}}=4 b^{2} H^{2}$.

It is clear that the warp factor decays and asymptotically disappears along the extra dimension, while the tachyon scalar possesses a kink or anti kink-like profile. Moreover, since the real tachyonic field has a bounded domain so the selfinteraction potential $V(T)$ which expressed in terms of the tachyonic scalar field $\mathrm{T}$ is a real and bounded potential. Also, it should be mentioned that as discussed in Ref. [15] this braneworld scenario is classically stable and capable of localizing gravity. It includes a graviton spectrum with a single massless bound state that corresponds to $4 D$ gravity localized on the brane and has a mass gap between the zero mode and massive modes that makes the $5 D$ corrections to Newton's and Coulomb's laws decay exponentially. Further discussions of this model were presented in $[31,45,46]$, where it was shown that it is possible to localize gauge, scalar and fermion fields in dS tachyonic braneworld. The localization of Elko spinor field on this brane may be very different from the ones of other matter fields hence we are interested to investigate this localization problem in the next section.

\section{Localization of Elko spinor fields with Yukawa-like couplings}

Elko is a new matter field which can be used to investigate some cosmological problems such as the horizon problem, the dark energy problem. It will be interesting to know whether a higher dimensional Elko field can be localized on various kinds of branes. The localization of the zero mode 
of a $5 D$ Elko spinor fields on Minkowski branes has been investigated in Refs. [38-40] by using a Yukawa-type coupling term between the five dimensional Elko spinor and the background scalar field. Recently, the authors of Ref. [41] has been introduced a new non-minimal coupling mechanism can provide the possibility of localizing the Elko zero mode on Minkowski branes. Since de Sitter and Anti-de Sitter branes have different properties from Minkowski branes thus localization of $5 D$ Elko field on these brane has new results compared to those on Minkowski branes. Localization of five-dimensional Elko spinors on dS/AdS thick branes has bees considered in Refs. [42,43]. The authors of [42] have found that the massless Elko field can be localized on the de Sitter brane with the non-minimal coupling mechanism whereas in Ref. [43] it was shown that the localization of the Elko zero mode on de Sitter and Anti-de Sitter thick branes can be realized with both mentioned localization mechanisms. In this section, we study the localization of Elko spinors on de Sitter tachyonic thick braneworld model generated by a tachyon scalar field by using the Yukawa-type coupling mechanism. Let us consider the action of an Elko spinor field $\lambda$ which is coupled to gravity as [38]

$$
\begin{aligned}
S= & \int d^{5} x \sqrt{-g}\left[-\frac{1}{4} g^{M N}\left(D_{M} \lambda D^{M} \bar{\lambda}\right.\right. \\
& \left.\left.+D_{M} \bar{\lambda} D^{M} \lambda\right)-\eta F(w) \lambda \bar{\lambda}\right],
\end{aligned}
$$

where $\eta$ is the coupling constant and $F(w)$ is a general scalar function of the extra dimensional coordinate $w$. The covariant derivatives are also given by

$$
D_{M} \lambda=\partial_{M} \lambda+\Omega_{M} \lambda, \quad D_{M} \bar{\lambda}=\partial_{M} \bar{\lambda}-\bar{\lambda} \Omega_{M} .
$$

The non-vanishing components of the spin connection $\Omega_{M}$ are [43]

$\Omega_{\mu}=\frac{1}{2}\left(\partial_{z} A\right) \gamma_{\mu} \gamma_{5}+\hat{\Omega}_{\mu}$

where $\mu, v, \bar{\mu}, \bar{v}=0,1,2,3$ and the $\gamma_{\mu}$ and $\gamma_{5}$ are the fourdimensional gamma matrixes on the brane and the $\gamma_{\mu}$ satisfy $\gamma_{\mu}, \gamma_{\nu}=2 \hat{g}_{\mu \nu}$, and $\hat{\Omega}_{\mu}$ is the spin connection on the brane. From the action (8) with the metric (1) and using the non-vanishing components of the spin connection (10), the equation of motion for the Elko spinor field can be expressed as

$$
\frac{1}{\sqrt{-g}} D_{M}\left(\sqrt{-g} g^{M N} D_{N} \lambda\right)-2 \eta F(w) \lambda=0
$$

In order to solve the above equation, as was proposed in Ref. [38], we use a KK decomposition of five-dimensional Elko spinor field as $\lambda=\lambda_{+}+\lambda_{-}$with

$$
\lambda_{ \pm}=e^{-\frac{3 A}{2}} \sum_{n}\left[\alpha_{n}(w) \varsigma_{ \pm}^{n}(x)+\alpha_{n}(w) \tau_{ \pm}^{n}(x)\right]
$$

where $\zeta_{ \pm}^{n}(x)$ and $\tau_{ \pm}^{n}(x)$ are two linear independent fourdimensional (4D) Elko spinor fields which satisfy

$$
\begin{aligned}
& \gamma^{\mu}\left(\partial_{\mu}+\hat{\Omega}_{\mu}\right) \varsigma_{ \pm}^{n}(x)=\mp i \varsigma_{\mp}^{n}(x), \\
& \gamma^{\mu}\left(\partial_{\mu}+\hat{\Omega}_{\mu}\right) \tau_{ \pm}^{n}(x)= \pm i \tau_{\mp}^{n}(x) . \\
& \gamma^{5} \varsigma_{ \pm}^{n}(x)= \pm \tau_{\mp}^{n}(x), \quad \gamma^{5} \tau_{ \pm}^{n}(x)=\mp \varsigma_{\mp}^{n}(x) \\
& \left(\partial^{\mu}+\hat{\Omega}^{\mu}\right)\left(\partial_{\mu}+\hat{\Omega}_{\mu}\right) \varsigma_{ \pm}^{n}(x)=m_{n}^{2} \varsigma_{ \pm}^{n}(x), \\
& \left(\partial^{\mu}+\hat{\Omega}^{\mu}\right)\left(\partial_{\mu}+\hat{\Omega}_{\mu}\right) \tau_{ \pm}^{n}(x)=m_{n}^{2} \tau_{ \pm}^{n}(x)
\end{aligned}
$$

Where $m_{n}$ is the mass of Elko spinor field on the brane. Also the KK modes $\alpha_{n}(w)$ satisfy the following equation of motion

$\dot{\alpha}_{n}-\left[\frac{3}{2} \hat{A}+\frac{13}{4} \AA^{2}+2 \eta e^{2 A} F(w)-m_{n}^{2}+i m_{n} \dot{A}\right] \alpha_{n}=0$

Where the prime denotes derivative with respect to extra dimension coordinate $w$. Furthermore, in order to getting the effective action on the brane for the four-dimensional massless Elko spinor field, we need the following orthonormality condition for the KK modes $\alpha_{n}(w)$ [38]

$\int_{-\infty}^{+\infty} \alpha_{n} \alpha_{n} d w=\delta_{m n}$

We now focus on the localization of the zero mode of a $5 D$ Elko spinor on de Sitter tachyonic branes. For the zero mode, i.e., the $4 D$ massless Elko spinor field, by setting $m_{n}=0$ in Eq. (16) the following schrödinger-like equation can be easily obtained

$\left[-\partial_{w}^{2}+V_{0}(w)\right] \alpha_{0}(w)=0$,

where the effective potential is given by

$V_{0}(w)=\frac{3}{2} \hat{A}+\frac{13}{4} \AA^{2}+2 \eta e^{2 A} F(w)$,

The potential (19) depends on the warp factor exponent $A(w)$ and the function $F(w)$. Because of existing freedom in the form of the Yukawa coupling one can include different forms of the function $F(w)$. In Refs. [38,39] this function is made of a background scalar field while in Ref. [40], it was assumed to be a function of Ricci scalar $R$. Furthermore, in Refs. [38,43] it was shown that by using similarities between the Elko field and the scalar field, the form of $F(w)$ in the Yukawa-type coupling mechanism is determined by the warped factor which is arising from the regulation of coefficient numbers of $A^{\prime}$ and $A^{2}$ in the effective potential of Schrodinger-like equation of Elko spinor field. This specific choice of the function $F(w)$ leads to the zero mode solution with the exponential form that can be localized on dS/AdS braneworld models. We notice that in their formalism, the $F(w)$ is a function of extra dimension coordinate in thick braneworld model and has a constant value in the RSII thin braneworld model. While in 
our study in this section, we have solved the Schrodinger-like equation of massive and massless Elko spinor field without any restriction on the effective potential as well as the Yukawa coupling term similar to the one discussed in Ref. [45] for the scalar field localization on the tachyonic de sitter brane. In this work, we first consider two simple choices for the $F(w)$ such that the function $F(w)$ has a zero value and a non-zero constant value which corresponds to massless and massive Elko spinor fields respectively. Next, we investigate Elko field localization on tachyonic de Sitter brane by demanding the function $F(w)$ to be a function of tachyon scalar field.

\subsection{Case I: $F(w)=0$}

In this case we will consider a simple function $F(w)=0$ in the action (8) that describes a $5 D$ free massless Elko spinor field. For this case, by using the solution (4) the effective potential (19) can be rewritten as

$V_{0}(w)=\frac{1}{4} H^{2}\left[13-25 \operatorname{sech}^{2}(2 H w)\right]$,

where we have sat $c=0$ for simplicity. Considering the behavior of the above effective potential, we can study the localization of the zero mode of the massless Elko spinor field on the de Sitter tachyonic branes. In order to get a localized zero mode, the value of the effective potential at its minima must be negative. one can easily find that the effective potential (20) has a negative minimum $-3 H^{2}$ at $z=0$ and a positive maximum $\frac{13}{4} H^{2}$ at $z=\infty$ which mean that this potential has the shape of the well known modified PöschlTeller one and the zero mode of massless Elko spinor field may be trapped by this potential. After some calculations we can rewrite the Eq. (18) with the potential (20) as :

$\left[-\partial_{\bar{w}}^{2}-\frac{25}{16} \operatorname{sech}^{2}(\bar{w})\right] \alpha_{0}(\bar{w})=-\frac{13}{16} \alpha_{0}(\bar{w})$,

where we have used $\bar{w}=2 H w$. The zero mode of massless Elko spinor, $\alpha_{0}(\bar{w})$, reads

$\alpha_{0}(\bar{w})=c_{1} P_{v}^{\eta}(\tanh (\bar{w}))+c_{2} Q_{v}^{\eta}(\tanh (\bar{w}))$,

where $c_{1}, c_{2}$ are integral parameters, $\mathrm{P}$ and $\mathrm{Q}$ represent the first and second associated Legendre functions respectively. The degree $v$ and order $\eta$ are

$\eta=\frac{\sqrt{13}}{4}, \quad v=-\frac{1}{2}+\frac{\sqrt{29}}{4}$.

In order to get a localized mode the solution (22) should be vanish at infinity. Considering the properties of the associated legendre functions expressed in Refs. [38,42], one can easily find that the solution $\alpha_{0}(\bar{w})$ cannot converge at $w \rightarrow \pm \infty$ for these values of $\eta$ and $v$ given by Eq. (39). Thus we conclude that the free massless Elko spinor field can not be localized on de Sitter tachyonic branes. By following the procedure of Refs. [41,42] we have examined localization of massless Elko fields on de Sitter tachyonic branes with non minimal coupling mechanism by choosing two different forms of the auxiliary function $K(w)=0$ and $K(w)=\tanh (w)$. Results are similar to the ones obtained in the case of de Sitter brane in Ref. [42]. We have found that by setting $p=\frac{1}{2}$ in the results of Ref. [42] free massless Elko spinor field localization can be resorted in our braneworld model.

\subsection{Case II: $F(w)=M_{E l k o}^{2}$}

Here, in contrast to the Ref. [43], we have especially assumed that the Yukawa coupling function $F(w)$ has a non zero constant value. Therefore, we expect the zero mode solution of our model be different from those results. We now study the localization of bulk massive Elko spinor fields on the above presented tachyonic de Sitter thick braneworld by setting $F(w)=M_{E l k o}^{2}$ where $M_{E l k o}$ is the $5 D$ Elko mass. With this choice for the function $F(w)$ the effective potential (19) of massive Elko field is given by

$$
\begin{aligned}
V_{0}(w)= & \frac{1}{4} H^{2}\left[13-25 \operatorname{sech}^{2}(2 H w)\right] \\
& +2 \eta \mathbf{s} M_{E l k o}^{2} \operatorname{sech}(2 H w),
\end{aligned}
$$

The values of this potential at $w=0$ and $w \rightarrow \pm \infty$ can be obtained as

$$
\begin{aligned}
& V_{0}(w=0)=-3 H^{2}+2 \eta \mathbf{s} M_{E l k o}^{2} \\
& V_{0}(w \rightarrow \pm \infty)=\frac{13}{4} H^{2} .
\end{aligned}
$$

Since the effective potential $V_{0}(w)$ tends to a constant value at the infinity so the asymptotic behavior shows that the potential is of pöschl-Teller type with a massless KK state localized on the brane. Also, the potential has a minimum at $w=0$ which is always negative for

$0<M_{E l k o}^{2}<-\frac{\kappa_{5}^{2} \Lambda_{5}}{4 \eta}$,

which indicates that the potential may trap the zero mode of a massive bulk Elko spinor field. The effective potential (24) is plotted in Fig. 1 for various values of $M_{\text {Elko }}^{2}$. From this figure, we can find that as $M_{E l k o}^{2}$ increases, the minimum of the potential tends to a zero value and then becomes positive when $-\frac{\kappa_{5}^{2} \Lambda_{5}}{4 \eta}<M_{E l k o}^{2}<-\frac{25 \kappa_{5}^{2} \Lambda_{5}}{24 \eta}$. Finally, when $M_{\text {Elko }}^{2}>$ $-\frac{25 \kappa_{5}^{2} \Lambda_{5}}{24 \eta}$ the potential has only a maximum in the origin, making evident that there are no localized modes at all.

In order to get the zero mode solution, by inspiring the process of Ref. [45] we use the following change of variables

$\bar{w}=\operatorname{arcsech}(u) \quad \alpha_{0}(\bar{w})=\operatorname{sech}^{\xi}(\bar{w}) \rho_{0}(\bar{w})$, 


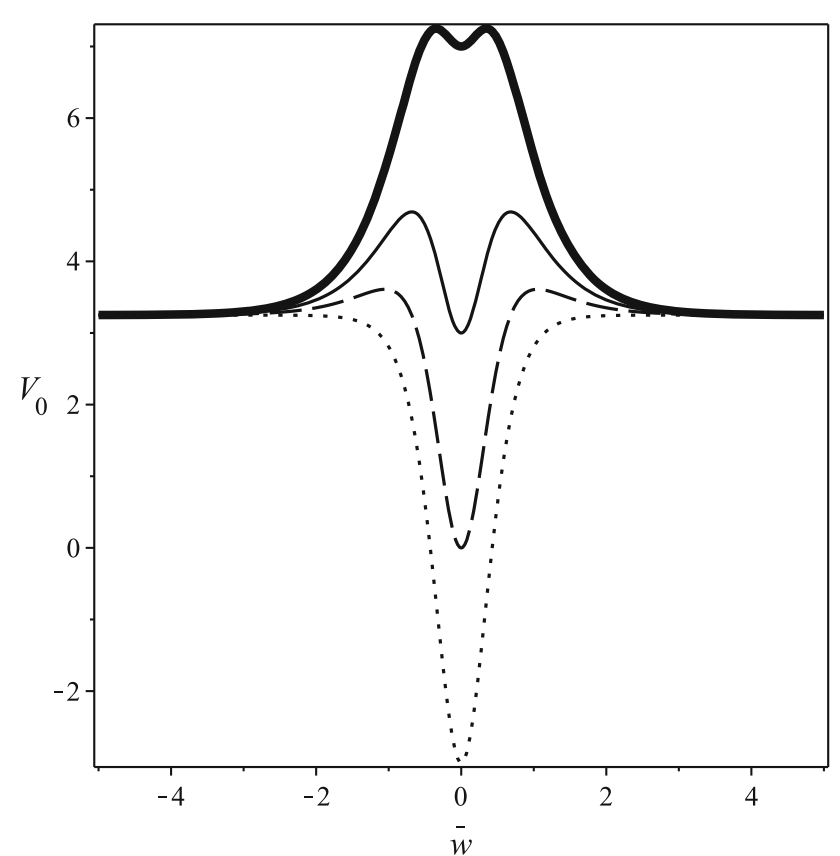

(a)

Fig. 1 The profile of the potential $V_{0}(w)$ for $M_{E l k o}^{2}=0$ (dotted line), $M_{\text {Elko }}^{2}=1.5$ (dashed line), $M_{\text {Elko }}^{2}=3$ (thin line), $M_{\text {Elko }}^{2}=5$ (thick line) and $\mathrm{s}, \mathrm{H}, \mathrm{b}=1$.

where $\xi=-\frac{1}{2}+\frac{\sqrt{29}}{4}$. Substituting Eqs. (24) and (27) in Eq. (18) and using $\bar{w}=2 H w$ we can get

$$
\begin{aligned}
& \frac{d^{2} \rho_{0}(\bar{w})}{d u^{2}}+\left[\frac{2(\xi+1) u^{2}-(2 \xi+1)}{u\left(u^{2}-1\right)}\right] \frac{d \rho_{0}(\bar{w})}{d u} \\
& +\left[\frac{\left.\frac{13}{16}+\frac{\eta \delta M_{E l k o}^{2} u-\xi^{2}}{2 H^{2}}\right]}{u^{2}\left(u^{2}-1\right)}\right] \rho_{0}(\bar{w})=0 .
\end{aligned}
$$

The corresponding solution for the localized zero mode of massive Elko field is given in terms of general Heun functions by solving Eq. (28) as

$$
\begin{aligned}
\alpha_{0}(\bar{w})= & \operatorname{sech}^{\xi}(\bar{w}) \rho_{0}(\bar{w}) \\
\propto & \operatorname{sech}^{\frac{\sqrt{13}}{4}}(\bar{w}) \sqrt{1-\operatorname{sech}(\bar{w})} H \operatorname{eun} G \\
& {\left[-1, a_{+}, b_{+}, c_{+}, d_{+}, \frac{1}{2},-\operatorname{sech}(\bar{w})\right] . }
\end{aligned}
$$

The coefficients of the solution (29) are given by

$$
\begin{aligned}
& a_{+}=\frac{\eta \mathbf{s} M_{E l k o}^{2}}{2 H^{2}}+\frac{d_{+}}{2}, \\
& b_{+}=1+\xi+\frac{d_{+}}{2} \\
& c_{+}=\frac{8 \xi d_{+}+4 \xi+6 d_{+}-25}{4+4 \sqrt{29}},
\end{aligned}
$$

where $d_{+}=1+\frac{\sqrt{13}}{2}$. Since the general Heun functions tend to the constant one at infinity [47], it is easy to find that the $\alpha_{0}(\bar{w}) \rightarrow e^{-\frac{\sqrt{13}}{4}|w|}$ as $w \rightarrow \pm \infty$. Thus the orthonormality condition $\int \alpha_{0}^{2} d w<\infty$ is satisfied that means the zero mode of massive Elko fields can be localized on the tachyonic de Sitter thick braneworld within the range of allowed values for the bulk mass of the Elko field, i.e. for $0<M_{E l k o}^{2}<-\frac{\kappa_{5}^{2} \Lambda_{5}}{4 \eta}$. We finally mention that if we follow the process of Ref. [43] and take

$$
\begin{aligned}
F(w)= & -\frac{H^{2}}{2 \eta s} \cosh (2 H w) \\
& \times\left[\left(\mathfrak{p}^{2}+\mathfrak{p}-25 / 4\right) \tanh ^{2}(2 H w)-2 \mathfrak{p}+3\right],
\end{aligned}
$$

then the zero mode solution is given by

$\alpha_{0}(w) \propto e^{\mathfrak{p} A(y)}=s^{\frac{\mathfrak{p}}{2}} \operatorname{sech}^{\frac{\mathfrak{p}}{2}}(2 H w)$

where $\mathfrak{p}$ is a real constant parameter that must be positive for a localized zero mode.

3.3 Case III: $F(w)=\frac{\sinh ^{2}\left(\frac{T}{b}\right)}{1-\sinh ^{2}\left(\frac{T}{b}\right)}$

In this subsection we examine Elko field localization by considering a tachyonic function $F(w)$ as the Yukawa interaction between Elko spinor field and background tachyonic scalar field. These types of functions have been used in Ref. [31] in order to study fermion field localization in a tachyonic de Sitter thick braneworld. With the choice of $F(w)$

$F(w)=\frac{\sinh ^{2}\left(\frac{T}{b}\right)}{1-\sinh ^{2}\left(\frac{T}{b}\right)}=\frac{\cosh (\bar{w})-1}{2}$,

the effective potential (19) can be rewritten as

$V_{0}(\bar{w})=\frac{1}{4} H^{2}\left[13-25 \operatorname{sech}^{2}(\bar{w})\right]+\eta \mathbf{s}\left(1-\operatorname{sech}^{2}(\bar{w})\right)$,

where we have used the solution (5) for the tachyon scalar field $T$. Also it is mentioned that this effective potential has the shape of the well known modified Pöschl-Teller one and the value of the potential at its minimum is always negative that means the zero mode of Elko field may be exist. Using definitions (27) and the above potential (36) in Eq. (18) one can easily found the following schrodinger-like equation

$$
\begin{aligned}
\frac{d^{2} \rho_{0}}{d u^{2}}+ & {\left[\frac{2(\xi+1) u^{2}-(2 \xi+1)}{u\left(u^{2}-1\right)}\right] \frac{d \rho_{0}}{d u} } \\
+ & {\left[\frac{\frac{13}{16}+\gamma(1-u)-\xi^{2}}{u^{2}\left(u^{2}-1\right)}\right] \rho_{0}=0, }
\end{aligned}
$$

that leads to following zero mode solution for the Elko spinor field 


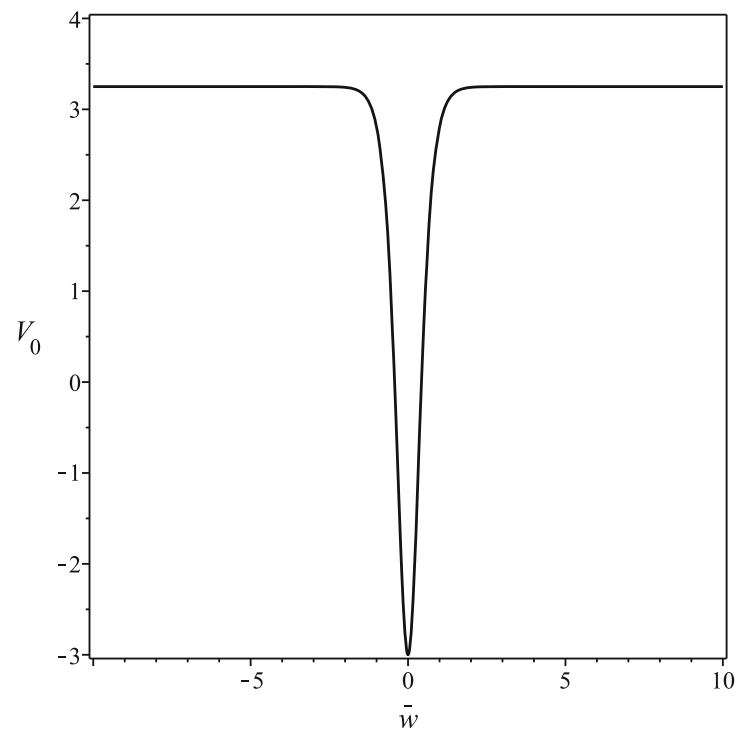

(a)

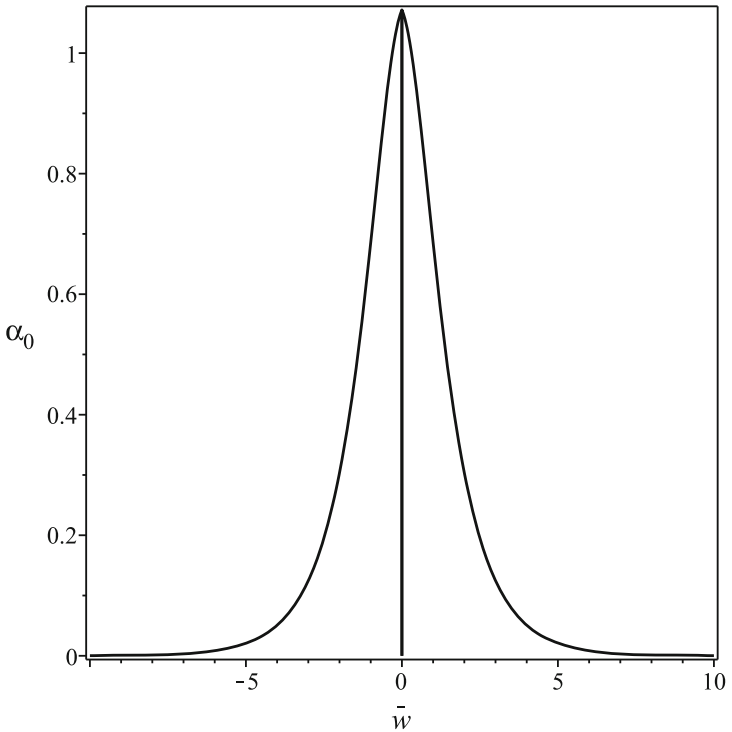

(b)

Fig. 2 The profile of a the potential $V_{0}(z)$ and $\mathbf{b}$ the zero mode $\alpha_{0}(\bar{w})$ for $b=1, H=1, \eta=0.005$

$$
\begin{aligned}
& \alpha_{0}(\bar{w}) \propto \operatorname{sech}^{\frac{\sqrt{13+16 \gamma}}{4}}(\bar{w}) \sqrt{1-\operatorname{sech}(\bar{w})} \text { Hеип } G \\
& \quad \times\left[-1, \tilde{a}_{+}, \tilde{b}_{-}, \tilde{b}_{+}, \tilde{d}_{+}, \frac{1}{2},-\operatorname{sech}(\bar{w})\right],
\end{aligned}
$$

where

$$
\begin{gathered}
\gamma=\frac{\eta \mathbf{s}}{4 H^{2}} \quad \tilde{d}_{+}=1+\frac{1}{2} \sqrt{13+16 \gamma}, \\
\tilde{a}_{+}=1-\gamma+\frac{\tilde{d}_{+}}{2} \quad \tilde{b}_{ \pm}=\frac{1}{2} \pm \frac{\sqrt{29}}{4}+\frac{\tilde{d}_{+}}{2}
\end{gathered}
$$

Since the general Heun function has only a constant contribution at infinity of the extra dimension, the zero mode $\alpha_{0}(\bar{w})$ tends to $e^{\frac{-\sqrt{13+16 \gamma}}{4}}|\bar{w}|$ az $\bar{w} \rightarrow \pm \infty$. Hence, it is clear that the orthonormality condition (17) is also satisfied, and the zero mode can be localized on the tachyonic de Sitter branes for any positive $\gamma$. The profile of the effective potential (36) and the zero mode (38) are shown in Fig. 2. As the Fig. 2b shows this kind of uncommon zero mode can be localized on the brane. We also mention that there exists a split at the point $w=0$ for the zero mode solution (38) as well as (29) which comes from the expression $(\sqrt{1-\operatorname{sech}(\bar{w})})$.

\section{Localization of Elko field with derivative coupling}

In this section, we study Elko spinor field localization on the tachyonic de Sitter brane by using a non-Yukawa derivative coupling term in the $5 d$ action. This mechanism was employed in order to solve the problem of fermion localization in thick braneworld models generated by an even background scalar field [28]. Also, the authors of Refs. [29,48] shown that fermion localization can be achieved on the Bloch branes and Deformed branes with the derivative coupling mechanisms. In this work, we are interested to know what happens when one tries to introduce a non-Yukawa coupling term in the bulk Elko field action. The five-dimensional action for a Elko spinor coupled to the background tachyon scalar fields with derivative coupling is

$$
\begin{aligned}
S= & \int d^{5} x \sqrt{-g}\left[-\frac{1}{4} g^{M N}\left(D_{M} \lambda D^{M} \bar{\lambda}+D_{M} \bar{\lambda} D^{M} \lambda\right)\right. \\
& \left.+\tilde{\eta} \bar{\lambda} \gamma^{M} \partial_{M} F(w) \gamma^{5} \lambda\right],
\end{aligned}
$$

where $\tilde{\eta}$ is a coupling constant. $\gamma^{M}=e_{\bar{M}}^{M} \gamma^{\bar{M}}$ where $\gamma^{\bar{M}}$ and $e_{\bar{M}}^{M}$ are used for the gamma matrix in flat space time and the vierbein respectively. These parameters satisfy the orthonormality relation $g_{M N}=e_{M}^{\bar{M}} e_{N}^{\bar{N}} \eta_{\bar{M}} \bar{N}$ and $\left\{\gamma^{M}, \gamma_{N}\right\}=2 \eta^{M N} \mathbb{I}$ with $\mathbb{I}=e_{\bar{v}}^{\mu} . \bar{M}, \bar{N}, \ldots=0,1,2,3,5$ stand for the five-dimensional local Lorentz indices and $\eta^{M N}=\operatorname{diag}(-,+,+,+,+)$. The equation of motion for the five-dimensional Elko spinor arising from the above action with the metric (1) is

$$
\frac{1}{\sqrt{-g}} D_{M}\left(\sqrt{-g} g^{M N} D_{N} \lambda\right)+2 \tilde{\eta} e^{A} \partial_{w} F(w) \lambda=0 .
$$

Using the decomposition (12) and the relations (13-15) for the Elko spinor $\lambda$, one can easily find that the massless zero mode satisfy the following schrödinger-like equation

$\left[-\partial_{w}^{2}+\mathbb{V}_{0}(w)\right] \alpha_{0}(w)=0$

where the effective potential is given by

$\mathbb{V}_{0}(w)=\frac{3}{2} \hat{A}+\frac{13}{4} \hat{A}^{2}-2 \tilde{\eta} e^{A} \partial_{w} F(w)$. 
We note that this effective potential is different from the one considered in the previous section for the usual Yukawa coupling. Also, it is well known that there exist many similarities between the Elko field and the scalar field $[38,43]$. The scalar effective potential with the form $V_{\phi}=\frac{3}{2} \hat{A}+\frac{9}{4} A^{2}$ is capable to trap the massless zero mode of scalar field while the five-dimensional free massless Elko spinor field can not be localized by the effective potential $V_{0}=\frac{3}{2} \hat{A}+\frac{13}{4} A^{2}$ because of existing an additional term $A^{2}$. When the coupling function $F(w)$ is used, the coefficient numbers of $A$ and $A^{2}$ can be adjusted as

$\frac{3}{2} \hat{A}+\frac{13}{4} \hat{A}^{2}-2 \tilde{\eta} e^{A} \partial_{w} F(w)=p \hat{A}+(p \hat{A})^{2}$.

where $p$ is a real parameter. It is clear that the equation (45) leads to the following solution for the function $F(w)$

$F(w)=-\frac{1}{2 \tilde{\eta}} \int e^{-A}\left[\left(p-\frac{3}{2}\right) \hat{A}+\left(p^{2}-\frac{13}{4}\right) \dot{A}^{2}\right]$,

that satisfy the condition $F(0)=0$. By substituting the Eq. (46) in the effective potential (44), one can easily find the Elko zero mode solution from the Eq. (43) which is $\alpha_{0}(w) \propto$ $e^{p A(w)}$. Next, we will investigate localization of Elko field on tachyonic de Sitter branes stated in section 2 by using the new derivative coupling mechanism presented here. Considering the solution (4) and recent discussions, the effective potential and the Elko zero mode solution are given by

$$
\begin{gathered}
\mathbb{V}_{0}(w)=p \hat{A}+(p \dot{A})^{2}=p^{2} H^{2} \tanh ^{2}(2 H w) \\
-2 p H^{2} \operatorname{sech}^{2}(2 H w), \\
\alpha_{0}(w) \propto e^{p A(w)}=\mathbf{s}^{\frac{p}{2}} \operatorname{sech}^{\frac{p}{2}}(2 H w) .
\end{gathered}
$$

It is easy to show that the zero mode (48) is normalizable for any positive $p$, so it is localized on the tachyionic ds brane. The profiles of the effective potential which is a PT potential and the zero mode are plotted in Fig. 3 for $\mathbf{s}=1, H=1 / 2$ and $p=1$.

\subsection{Example: $F(w)=-\frac{1}{H}$ Eliptic $F\left(\cosh \frac{\bar{w}}{2}, \sqrt{2}\right)$}

In the previous subsection, we have found that in order to obtain the Elko zero mode on a tachyonic de Sitter brane, the form of $F(w)$ in the derivative coupling mechanism must be determined by adjusting the coefficient of the $A$ in the effective potential (44) same as the one of the scalar case. Here, by an example it is shown that an appropriate form of $F(w)$ leads to the Elko zero mode localization on tachyonic dS brane without needing to regulate the coefficient of the effective potential. We assume that

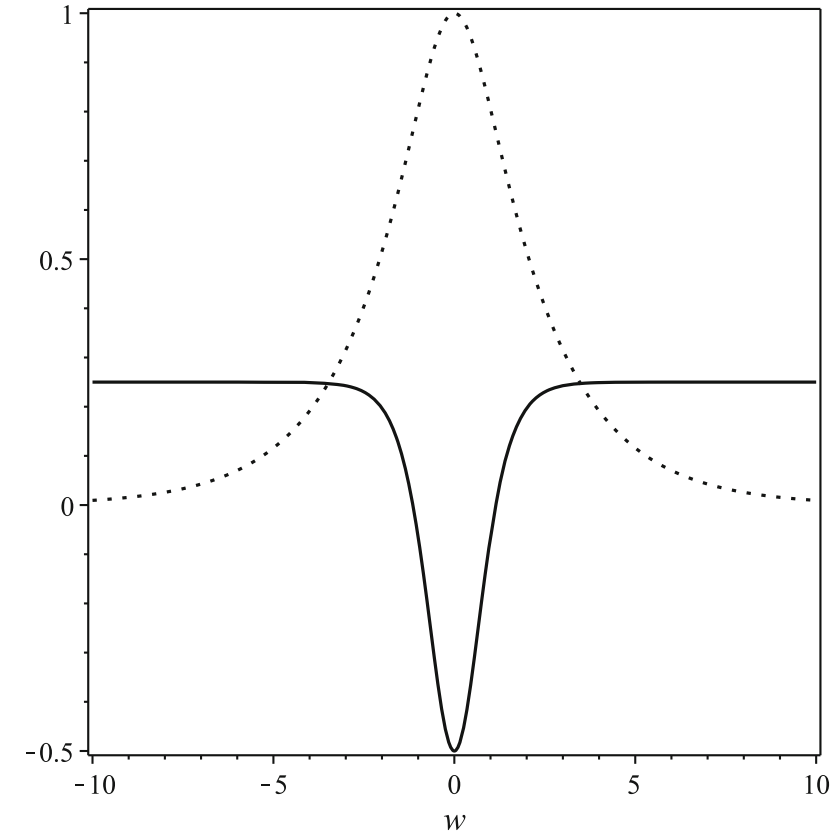

(a)

Fig. 3 The profile of the potential $V_{0}(z)$ (solid line) and the zero mode $\alpha_{0}(\bar{w})$ (dotted line) for $s=1, H=1 / 2, p=1$

$F(w)=-\frac{1}{H} \operatorname{Eliptic} F(\cosh (H w), \sqrt{2})$,

where ElipticF $(u, v)$ gives the elliptic integral of the first kind $F(u, v)$. Furthermore, the derivative of the function $F(w)$ is

$\partial_{w} F(w)=\frac{2}{\sqrt{2 \cosh ^{2}(H w)-1}}$.

Using above equation and relation (4) in the effective potential (44) we get

$\mathbb{V}_{0}(\bar{w})=\frac{1}{4} H^{2}\left[13-25 \operatorname{sech}^{2}(\bar{w})\right]-4 \tilde{\eta} \sqrt{\mathbf{s}} \operatorname{sech}(\bar{w})$.

It is clear that the effective potential (51) is a pöschl-Teller potential which always has a negative value at its minimum for positive values of $\tilde{\eta}$. Following the process explained in Sect. 3, the Elko zero mode solution can be obtained from Eq. (43) as

$$
\begin{aligned}
& \alpha_{0}(\bar{w}) \propto \operatorname{sech}^{\frac{\sqrt{13}}{4}}(\bar{w}) \sqrt{1-\operatorname{sech}(\bar{w})} \text { HeunG } \\
& \quad \times\left[-1, \frac{d_{+}}{2}-4 \tilde{\eta} \sqrt{\mathbf{s}}, b_{+}, c_{+}, d_{+}, \frac{1}{2},-\operatorname{sech}(\bar{w})\right],
\end{aligned}
$$

where the parameters $b_{+}$and $c_{+}$are same as Eqs. (31) and (32) respectively. We mention that the zero mode (52) is very similar to the localized one obtained in Sect. 3.2 for massive Elko spinor field. Hence we adopt more discussion about it 
and conclude that by choosing an adequate form of $F(w)$ the Elko field can also be localized on tachyonic de Sitter brane with derivative coupling mechanism.

\section{Conclusions}

In this work, we have investigated the localization of Elko spinor fields on a tachyonic de Sitter thick brane generated by a tachyon scalar field. Using the Yukawa-type coupling mechanism, we have analytically investigated the zero mode solution. For the case of massless Elko spinor field we have found that the zero mode was given in terms of associated Legendre functions which cannot be localized on tachyonic de Sitter brane. However, massive Elko spinor fields can only be localized on this brane when the bulk mass bounded as $0<M_{\text {Elko }}^{2}<-\frac{\kappa_{5}^{2} \Lambda_{5}}{4 \eta}$. Furthermore, we have studied the Elko field localization by considering a tachyonic function $F(T(w))$ as the Yukawa interaction between Elko spinor and background tachyonic scalar field. It was shown that the zero mode is given in terms of general Heun functions that its behavior at infinity is $e^{\frac{-\sqrt{13+16 \gamma}}{4}}|\bar{w}|$ which means such zero modes can be localized on the tachyonic de Sitter brane for any value of $\gamma$.

In order to localize the Elko spinor fields, following the Ref. [28] we have also applied a new mechanism i.e., the derivative coupling between the five-dimensional Elko spinor and the background tachyon scalar field. In this mechanism, the function $F(w)$ is determined by adjusting the coefficient of the $A$ in the effective potential and the zero mode of Elko spinor field has the form of $e^{p A}$ that is localized on the brane under the condition $p>0$. Finally, we focused on the case of $F(w)=-\frac{1}{H}$ Eliptic $F(\cosh (H w), \sqrt{2})$ and found that the Elko zero mode can be trapped on tachyonic dS brane without needing to regulate the coefficient of the effective potential. For both mechanisms, it was shown that the effective potential of Elko spinor zero mode in the corresponding Schrödinger equation is PT-like effective potential which is not applicable for studying the massive KK modes. It will be interesting to consider the excited modes of the theory. This and other related issues are presently under consideration and will be investigated in our further work.

Data Availability Statement This manuscript has no associated data or the data will not be deposited. [Authors' comment: The data that support the findings of this study are available from the corresponding author upon reasonable request.]

Open Access This article is licensed under a Creative Commons Attribution 4.0 International License, which permits use, sharing, adaptation, distribution and reproduction in any medium or format, as long as you give appropriate credit to the original author(s) and the source, provide a link to the Creative Commons licence, and indicate if changes were made. The images or other third party material in this article are included in the article's Creative Commons licence, unless indi- cated otherwise in a credit line to the material. If material is not included in the article's Creative Commons licence and your intended use is not permitted by statutory regulation or exceeds the permitted use, you will need to obtain permission directly from the copyright holder. To view a copy of this licence, visit http://creativecomm ons.org/licenses/by/4.0/.

Funded by $\mathrm{SCOAP}^{3}$.

\section{References}

1. V.A. Rubakov, M.E. Shaposhnikov, Phys. Lett. B 125, 139 (1983)

2. R. Linares, H.A. Morales-Tecotl, O. Pedraza, Phys. Rev. D 81, 126013 (2010)

3. M.N. Smolyakov, I.P. Volobuev, J. Exp. Theor. Phys 108, 597 (2009)

4. L. Randall, R. Sundrum, Phys. Rev. Lett. 83, 3370 (1999)

5. L. Randall, R. Sundrum, Phys. Rev. Lett. 83, 4690 (1999)

6. A. Kehagias, K. Tamvakis, Phys. Lett. B 504, 38 (2001)

7. H. Guo, Y.-X. Liu, Z.-H. Zhao, F.-W. Chen, Phys. Rev. D 85, $124033(2012)$

8. A.Z. Wang, Phys. Rev. D 66, 024024 (2002)

9. R.R. Caldwell, Phys. Lett. B 545, 23 (2002)

10. M. Minamitsuji, W. Naylor, M. Sasaki, Nucl. Phys. B 737, 121 (2006)

11. I. Olasagasti, K. Tamvakis, Phys. Rev. D 68, 064016 (2003)

12. A. Kehagias, K. Tamvakis, Phys. Lett. B 504, 38 (2001)

13. V. Kamali, E.N. Nik, Eur. Phys. J. C 77, 449 (2017)

14. D. Bazeia, F.A. Brito, J.R. Nascimento, Phys. Rev. D 68, 085007 (2003)

15. G. German, A. Herrera-Aguilar, D. Malagon-Morejon, R.R. MoraLuna, R. da Rocha, JCAP 1302, 035 (2013)

16. N. Barbosa-Cendejas, R. Cartas-Fuentevilla, A. Herrera-Aguilar, R.Rigel Mora-Luna, R. da Rocha, JCAP 01, 005 (2018)

17. B. Bajc, G. Gabadadze, Phys. Lett. B 474, 282 (2000)

18. J. Liang, Y.-S. Duan, Phys. Lett. B 678, 491 (2009)

19. H. Guo, Y.-X. Liu, S.-W. Wei, C.-E. Fu, Europhys. Lett 97, 60003 (2012)

20. A. Tofighi, M. Moazzen, Acta Phys. Polon. B 45, 1797 (2014)

21. M. Moazzen, Z. ghalenovi, Ann. Phys. 385, 70 (2017)

22. C.A. Vaquera-Araujo, O. Corradini, Eur. Phys. J. C 75, 48 (2015)

23. A.E.R. Chumbes, J.M.H. da Silva, M.B. Hott, Phys. Rev. D 85, 085003 (2012)

24. M. Moazzen, Int. J. Mod. Phys. A 32, 1750058 (2017)

25. A. Tofighi, M. Moazzen, A. Farokhtabar, Int. J. Theor. Phys. 55, $1105(2016)$

26. A. Tofighi, M. Moazzen, Int. J. Theor. Phys. 50, 1709 (2011)

27. L.B. Castro, L.E.Arroyo Meza, Europhys. Lett. 102, 21001 (2013)

28. Y.-X. Liu, Z.-G. Xu, F.-W. Chen, S.-W. Wei, Phys. Rev. D 89, 086001 (2014)

29. M.Moazzen sorkhi, Z. ghalenovi, Acta Phys. Polon. B 49, 123 (2018)

30. Y.-X. Liu, H. Guo, C.-E. Fu, H.-T. Li, Phys. Rev. D 84, 044033 (2011)

31. R.C. Fuentevilla, A. Escalante, G. German, A. Herrera-Aguilara, R.Rigel Mora-Luna, JCAP 05, 026 (2016)

32. Y.-X. Liu, L.-D. Zhang, S.-W. Wei, Y.-S. Duan, JHEP 08, 041 (2008)

33. W.T. Cruz, A.R. Gomes, C.A.S. Almeida, Eur. Phys. J. C 71, 1790 (2011)

34. MMoazzen sorkhi, E. Mazani, Mod. Phys. Lett. A 33(40), 1850235 (2018)

35. D.V. Ahluwalia, D. Grumiller, Phys. Rev. D 72, 067701 (2005)

36. D.V. Ahluwalia, D. Grumiller, JCAP 0507, 012 (2005)

37. D.V. Ahluwalia, C.Y. Lee, D. Schritt, Phys. Lett. B 687, 248 (2010) 
38. Y.X. Liu, X.N. Zhou, K. Yang, F.W. Chen, Phys. Rev. D 86, 064012 (2012)

39. I.C. Jardim, G. Alencar, R.R. Landim, R.N. Costa Filho, Phys. Rev. D 91(4), 048501 (2015)

40. I.C. Jardim, G. Alencar, R.R. Landim, R.N. Costa Filho, Phys. Rev. D 91(8), 085008 (2015)

41. X.N. Zhou, Y.Z. Du, Z.H. Zhao et al., Eur. Phys. J. C 78, 493 (2018)

42. M.Moazzen sorkhi, Z. ghalenovi, Int. J. Mod. Phys. A 33, 1850172 (2018)

43. X.-N. Zhou, Z.-H. Zhao, Y.-Z. Du, arXiv:1812.08332 [hep-th]

44. M. Moazzen Sorkhi, A. Tofighi, Z. Ghalenovi, Iran. J. Phys. Res. 19(3), 521-527 (2019)
45. A. Díaz-Furlong, A. Herrera-Aguilar, R. Linares et al., Gen. Relativ. Gravit. 46, 1815 (2014)

46. A. Herrera-Aguilar, A.D. Rojas, E. Santos-Rodriguez, Eur. Phys. J. C 74, 2770 (2014)

47. Y. S. Choun, arXiv:1303.0876v12

48. Q.-Y. Xie, H. Guo, Z.-H. Zhao, Y.-Z. Du, Y.-P. Zhang, Class. Quantum Gravity 34, 055007 (2017) 